\title{
Adapting the Ultrasonic/Sonic Driller/Corer for Walking/Climbing Robotic Applications
}

\author{
Mircea Badescu*, Xiaoqi Bao, Yoseph Bar-Cohen, Zensheu Chang, Borna E. Dabiri, Brett \\ Kennedy, Stewart Sherrit \\ JPL/Caltech, 4800 Oak Grove Drive, Pasadena, CA 91109-8099,
}

\begin{abstract}
Future NASA's missions include the search for past and existing life in the Universe and evidence on how the planets in the Solar system formed and evolved. In order to fulfill these goals sampling systems that meet the stringent requirements of the various environments are required to be developed. To support these objectives an ultrasonic/sonic driller/corer (USDC) device has been developed at Jet Propulsion Laboratory (JPL) to allow drilling and coring rocks for in-situ planetary analysis [1]. The site location and method of sampling are of vital importance to scientists. Surface rocks abrasion, small depth soil drilling, and deep drilling have been proposed. It has been suggested that another possible source of mineralogical or astrobiological information can be found by exploring the sidewall of canyons. The exploration of such sites requires the development of a limbed robotic system capable of walking and climbing slopes up to and including vertical faces and overhangs. An anchor/drilling mechanism is currently under development and is being installed on each leg of the four-legged Steep Terrain Access Robot (STAR). This paper presents the modeling, design, and preliminary testing results of the USDC for use as end-effectors of walking/climbing robots.
\end{abstract}

Keywords: Ultrasonic drilling, limbed robot, soil drill, piezoelectric devices.

\section{INTRODUCTION}

In-situ sampling and analysis, and possibly the sample return to earth are becoming increasingly important in NASA's space exploration missions. Moreover, to extract the full science potential from surface operations the sampling should be done over as diverse areas as possible on the surface of a planetary body. This suggests, robots, as sampling and analysis instruments carriers, must be able to access the entire surface of the planetary body. Indeed, the National Research Council (NRC) Decadal Report lists the slopes of the Moon's Aitkin Crater in particular and "hard-to-reach access" in general as priority targets for exploration.

In addition, existing drilling techniques are limited by the need for large axial forces and holding torques, high power consumption, large mass and inability to efficiently duty cycle. JPL's NDEAA group developed in collaboration with Cybersonics, Inc. a novel USDC mechanism [Bar-Cohen et al, 2001] specifically to address these issues.

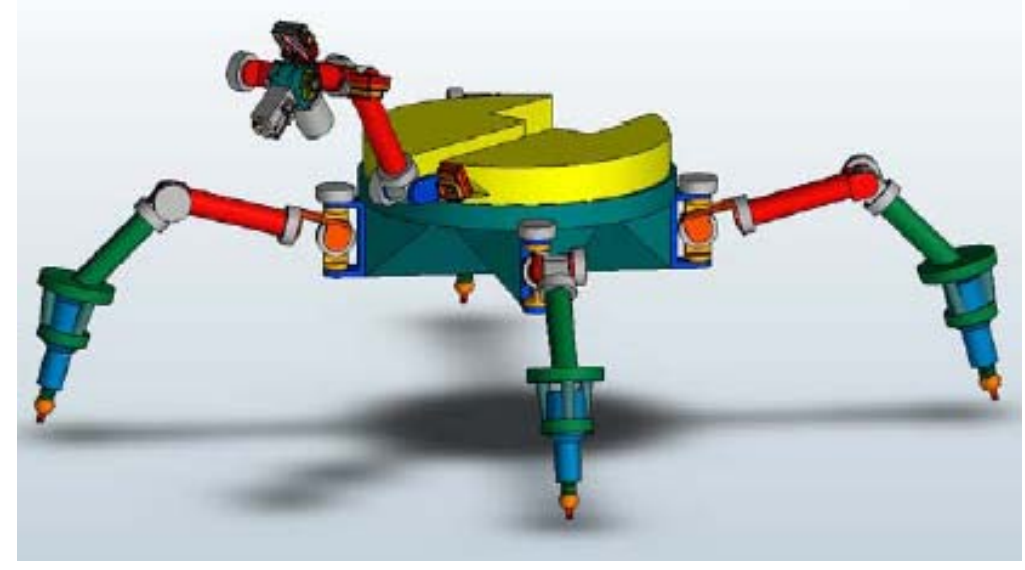

Figure 1 - STAR concept

In order to increase both sample access and sampling ability a JPL team proposed to design and build a limbed robotic system that will climb slopes up to and including vertical faces and overhangs, and be able to react forces to maintain

* Mircea.Badescu@jpl.nasa.gov; Phone 818-393-5700, Fax 818-393-4057; http://ndeaa.jpl.nasa.gov 
stability and do useful work (e.g., sample acquisition/instrument placement). Fig. 1 shows a concept model of the proposed platform. This capability will be enabled by the development of a new class of USDC end-effectors as well as the sensing and algorithms to robustly utilize them. The system is designed to allow both ascent and decent of steep walls through the use of temporary anchors. Although implicitly designed for space exploration these developments will also be benefit for terrestrial applications such as military scouts, search-and-rescue, and remote science collection. In the first part of this project the STAR team focused on USDC development, design changes of the USDC to respond to new climbing capability requirement, and software development. The first two focus areas are presented in this paper as theoretical findings and testing results.

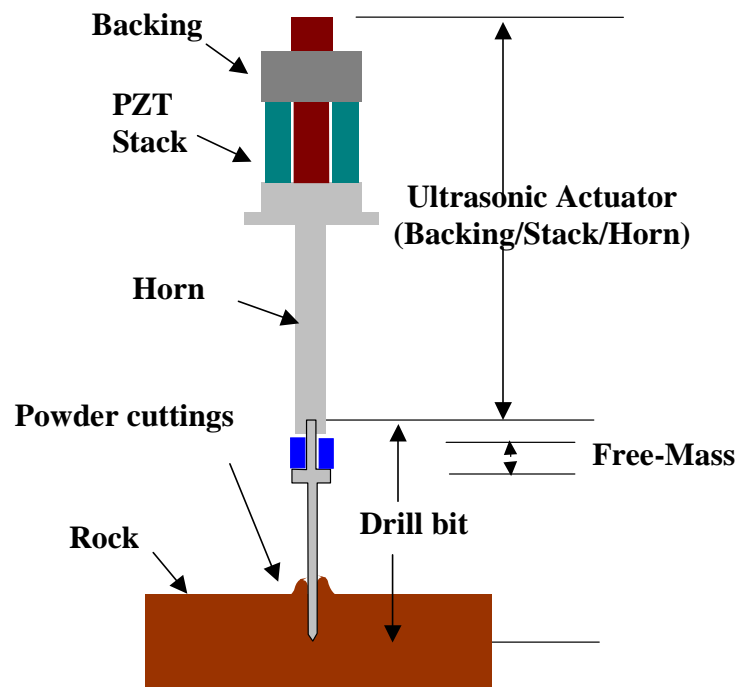

Figure 2 - USDC schematics

The USDC device, shown in Fig. 2, is based on an ultrasonic horn actuated by a piezoelectric stack, which impacts a free-mass resonating between the horn and a drill stem. The ultrasonic horn is driven at its resonance frequency, ranging from $5 \mathrm{KHz}$ to $25 \mathrm{KHz}$ depending on the specific design. The free mass bounces back and forth between the ultrasonic horn and the drill stem, converting the high frequency vibration of the horn to a hammering action with a frequency range of tens to a thousand Hz. This novel drill is capable of high-speed drilling using low axial preload and low power, and it is highly tolerant to misalignment. The USDC was demonstrated to operate from such robotic platforms as the Sojourner rover and the FIDO rover robotic arm and it has been shown to drill rocks as hard as granite and basalt and as soft as sandstone and tuff. It has drilled 25-mm (1-inch) deep holes in granite from a 4-kg platform, 15-cm deep and 3-mm diameter in sandstone and $10 \mathrm{~cm}$ long, $1 \mathrm{~cm}$ diameter cores of basalt. The USDC device is highly tolerant to changes in its operating environment, since it is driven by piezoelectric ceramics, which can be designed to operate over a wide range of temperatures including those expected on Mars as well as Venus. In Fig. 3, the USDC is shown being held from its power cord while drilling into sandstone rock -- this is possible because relatively low axial preload is required.

Robotic systems that compare to the proposed system fall into three distinct areas: gravity-stabilized legged and wheeled robots, tethered robots, and robots that adhere to the substrate. The first category is best represented by Mars Exploration Rovers at JPL and NOMAD [Wettergreen, 1997] and DANTE I [Wettergreen, 1993] (both Carnegie Mellon University) outside of JPL. The second category is characterized by the Cliffbot [Pirjanian et al, 2002] system at JPL and DANTE II [Bares and Wettergreen, 1999]. The third category covers MACS [Bar-Cohen and Backes, 2000] at JPL and the Gecko robots at iRobot.

The robots in each of these categories cover some portion of the operational envelope of the STAR concept. In the first case, the gravity-stabilized robots can traverse over rough terrain. However, these vehicles become unstable on slopes on which gravity becomes a destabilizing factor, approximately 45-55 degrees from horizontal.

The tethered systems overcome this limitation by counteracting the destabilizing force of gravity with tethers attached to anchors at the top of the slope. This solution itself creates a limitation due to the fact that the traverse must begin at the top of the slope. In addition, the tethered robot may loose contact with the substrate (and thus a level of controllability) when an overhang is encountered. An encounter with an overhang may also result in a non-reversible 
path. The third category relies on active or passive adhesion of the robot to the substrate, generally performed by suction cups, magnets, or sticky adhesives. Each of these adhesion methods applies to a very narrow range of substrate properties, primarily smooth, clean, non-friable surfaces or ferrous in the case of magnetics. While these techniques are useful for certain scenarios, those scenarios do not as a rule exist for space exploration.

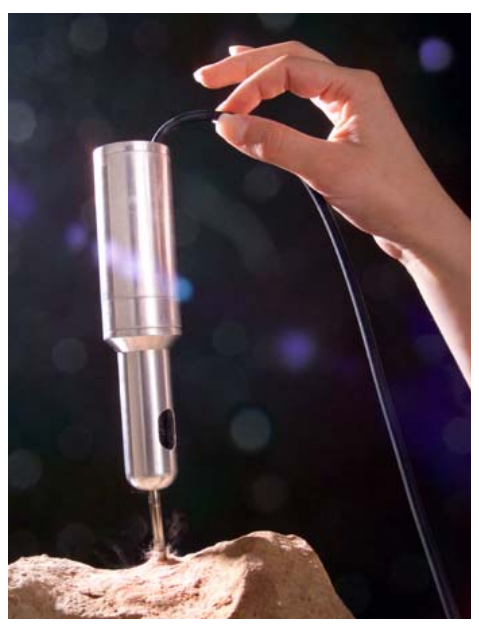

Figure 3 - USDC

The creation of a robotic system that will actively cling to vertical or overhanging slopes (i.e., achieve nonfrictional force closure relative to the terrain) requires three areas of development: an end-effector capable of creating footholds in various substrates, a robotic platform with sufficient dexterity to properly place those end-effectors, and the forcecontrol and planning algorithms necessary to direct the platform's actuators. Due to the advantages of the USDC (viz., simple design, low normal force during operation, dual use for mobility and sampling, and the potential for embedded instrumentation), this apparatus was chosen as the basis of the end-effector design.

Moreover, the design was improved to include active retraction to reduce the risk of jamming in the foothold. In addition, the USDC design was improved to also provide anchoring methods for loose terrain.

\section{MODELING}

The USDC consists of three major components, the ultrasonic actuator, the free mass, and the drill stem. The ultrasonic actuator is made of a stack of piezoelectric rings, an ultrasonic horn, a metal backing, and a pre-stress bolt to connect all these parts and provide pre-strain to the piezoelectric stack (Fig. 2). The piezoelectric material needs to be prestrained so it doesn't break during the extension. The stack of piezoelectric material is excited at the resonance frequency of the ultrasonic actuator. Through the amplification of the ultrasonic horn, the displacement of the vibration reaches tens of microns at the tip of the horn. A free mass is contacted to the horn tip of the USDC. During a drilling operation, the free mass moves back and forth between the ultrasonic horn and the drill stem. Due to the fact that the velocity of the free mass is smaller than the velocity of the horn tip vibration, the free mass usually contacts the horn tip at a favorable phase of horn tip vibration. During the collision the free mass picks up momentum and is accelerated back to the drill stem. The free-mass transfers impact momentum from the ultrasonic transducer to the drill stem at a frequency ranging from tens of $\mathrm{Hz}$ to about $1000 \mathrm{~Hz}$. The shock waves caused by the impacts of the free mass upon the drill stem propagate to the bit/rock interface. The impacted brittle medium (rock, ice, etc.) is fractured when its ultimate strain is exceeded at the medium/bit interface.

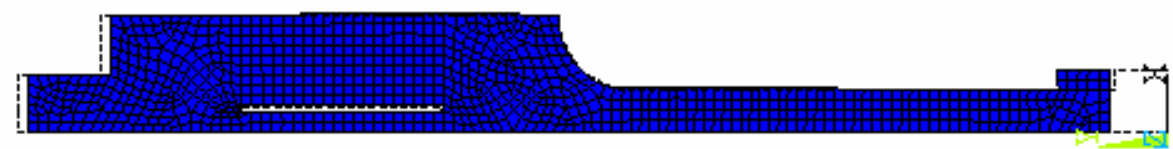

Figure 4 - Transducer - modal nalysis 
The modeling of the USDC has been done previously and presented in other publications [Sherrit et al, 2000; Bao et al, 2003; Sherrit et al, 2004]. ANSYS ${ }^{\mathrm{TM}}$ was used for modal and harmonic analysis of the transducer. Fig. 4 shows the the relative displacement of the horn tip (right) and the backing (left). The design was made such that the mounting point coincides with the neutral plane and so it has the lowest displacement. The specific USDC design for the Steep Terrain Access Robot (STAR) is presented in the next section.

\section{DESIGN}

The STAR USDC has three main functions: drill a hole in the climbing substrate while retaining the bit and avoid being stuck in the drilled hole, work as an anchor for the drilled hole, and work as an anchor in loose material.

The capability of the USDC to drill rocks and extract cores and powder has been previously demonstrated. The ability of the USDC design for this task is to hammer out of a hole in the event that the bit gets stuck. This requirement forced the development of new technology in the form of an integrated dual free mass (see Fig. 5) along with a "dog bone" actuator horn (see Fig. 6).

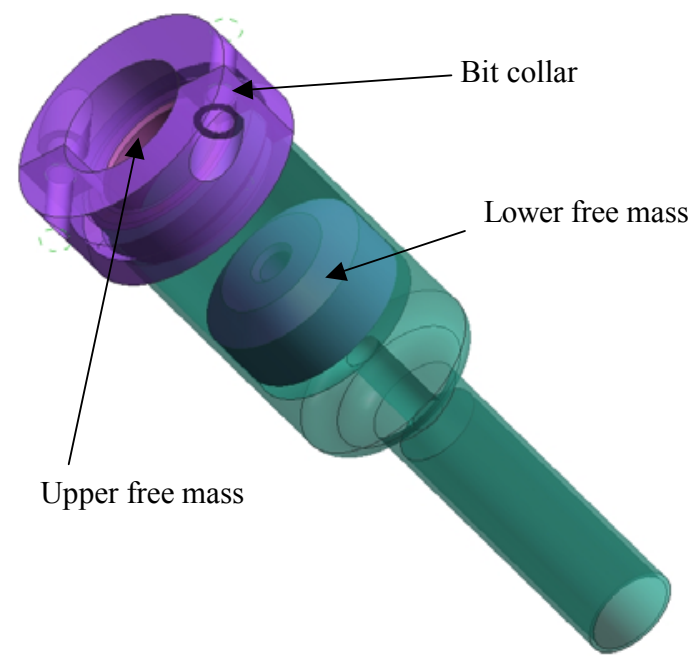

Figure 5 - Drill bit with integrated dual free mass

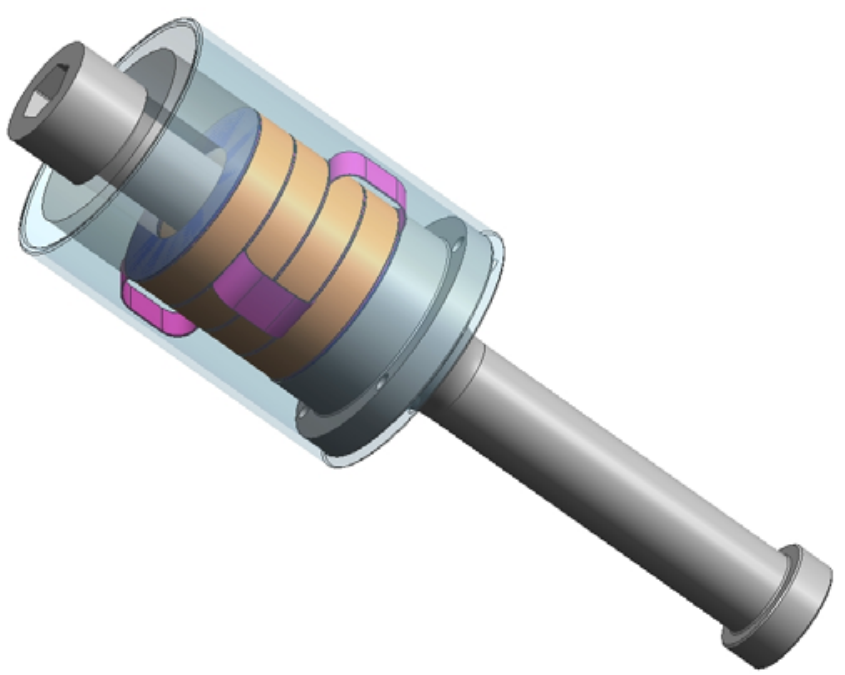

Figure 6 - USDC transducer with "dog bone" horn design 
The improvement allowed for a bit design with forward and reverse hammering action. This forward and reverse hammering and anchoring capability of the USDC is currently being patented by NASA. The final actuator design, shown without housing in Fig. 6, was developed through a series of iterations with the FEM models for the USDC transducer. The actuator is based on the "Dog bone" horn. At present the USDC does not accommodate core shearing and core retaining. Bit designs that enable these features are currently being contemplated. The capability of the USDC to be used as an anchor for the drilled hole implied designing the horn and the drill bit to be able to support the bending moment on the USDC when the whole weight of the STAR robot is supported by one leg on a vertical wall (worst case scenario). The depth of the hole to be drilled depends on the drilling material properties and load capacity of the USDC. The capability of the USDC to work as an anchor in loose material implies an increased transverse cross section area while maintaining the weight of the device at a low value. Also the axial cross section area is to be maintained at a minimum to allow the USDC to penetrate the loose material but stop at a certain depth. An additional competing requirement is the mass of the drill bit is to be maintained at a minimum for drilling efficiency. The solution chosen was to extend the housing (see Fig. 7) to cover the horn and part of the bit and have thin planar fins. Two sets of fins were introduced. A first set of four thin fins includes the USDC central axis. The fins are spaced at $90^{\circ}$. Another set forms a disk perpendicular on the central axis and limits the anchor penetration depth.

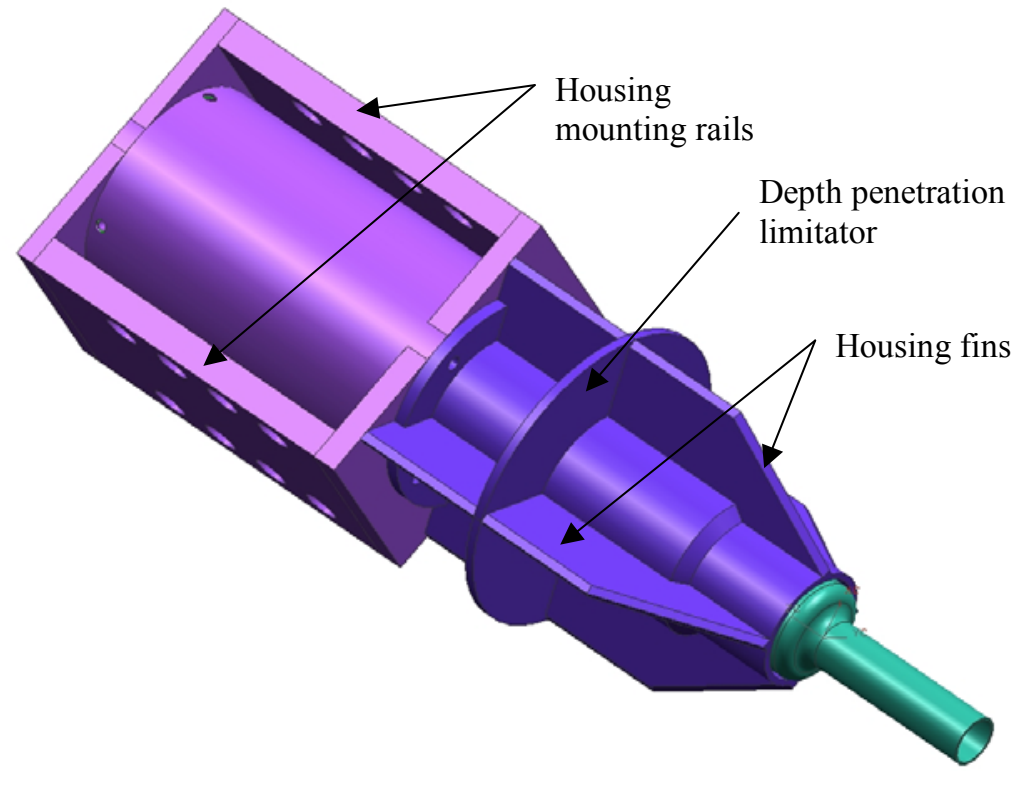

Figure 7 - USDC housing

\section{TESTING RESULTS}

Three sets of tests that involved the USDC as the end-effector of the robot limbs were performed during the first phase of the research: use of the USDC as anchors, loose material penetration study, and power consumption and drilling rates for the new USDC design. 


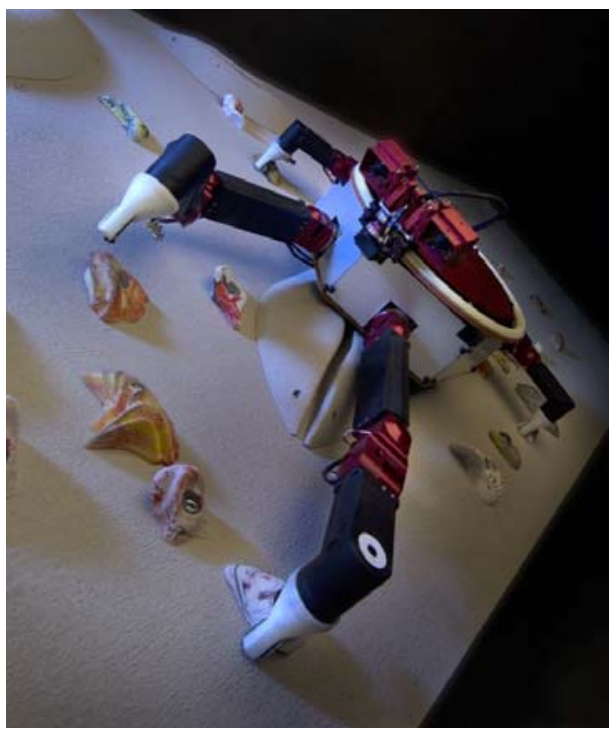

Figure 8 - STAR prototype

Prior to the prototype design an analysis of the required bit properties was conducted along with experimental tests to confirm the analysis. The two primary functions of the USDC bit for this task is to drill or core efficiently into a sedimentary type rock and to sustain load cases similar to those imposed by LEMUR limb shown in Fig. 8 performing STAR mobility operations.

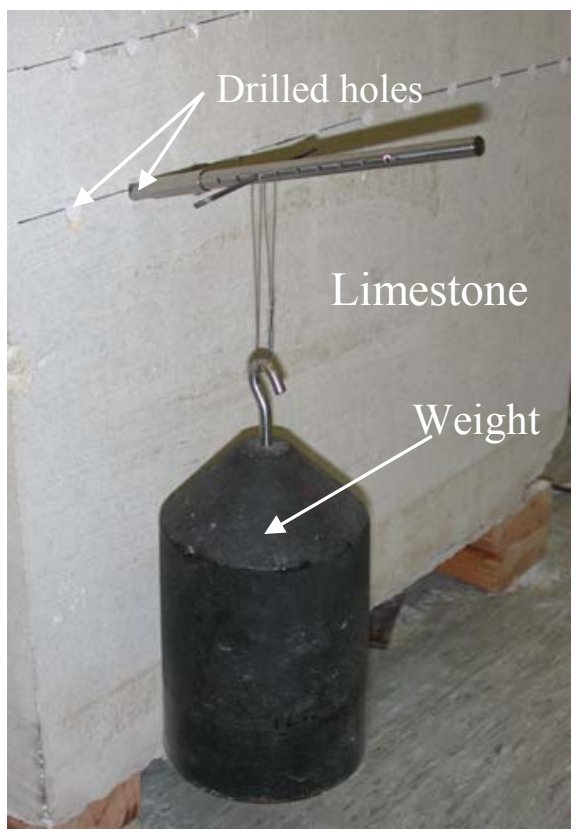

Figure 9 - Limestone testing setup

The primary bit design feature that allows for efficient drilling/coring of the USDC is a minimal cross sectional area, while the bending moment requirement however suggests a large area moment of inertia. These requirements were competitive and an optimal design was determined to accommodate both requirements. 
Bending moment $[\mathrm{N}-\mathrm{mm}]^{*}$

\begin{tabular}{|c|c|c|c|c|c|c|c|c|c|c|c|c|}
\hline \multicolumn{3}{|c|}{ Hole I Weight Location [mm] } & 75 & 89 & 103 & 117 & 131 & 145 & 159 & 173 & 187 & 201 \\
\hline No & Depth [mm] $]^{\star \star}$ & 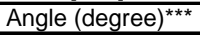 & & & & & & & & & & \\
\hline 1 & 17.0 & 25 & 5,848 & 7,259 & 8,671 & 10,082 & 11,494 & 12,905 & 14,317 & 15,728 & 17,140 & 18,551 \\
\hline 2 & 17.0 & 25 & 5,848 & 7,259 & 8,671 & 10,082 & 11,494 & 12,905 & 14,317 & 15,728 & & \\
\hline 3 & 17.0 & 25 & 5,848 & 7,259 & 8,671 & 10,082 & 11,494 & 12,905 & 14,317 & 15,728 & & \\
\hline 4 & 17.0 & 25 & 5,848 & 7,259 & 8,671 & 10,082 & 11,494 & 12,905 & 14,317 & 15,728 & & \\
\hline 5 & 17.0 & 25 & 5,848 & 7,259 & 8,671 & 10,082 & 11,494 & 12,905 & 14,317 & 15,728 & & \\
\hline 6 & 17.0 & 25 & 5,848 & 7,259 & 8,671 & 10,082 & 11,494 & 12,905 & 14,317 & 15,728 & & \\
\hline 7 & 17.0 & 20 & 6,063 & 7,527 & 8,990 & 10,454 & 11,917 & 13,381 & 14,844 & 16,308 & & \\
\hline 8 & 17.0 & 22 & 5,982 & 7,426 & 8,870 & 10,314 & 11,759 & 13,203 & 14,647 & 16,091 & & \\
\hline 9 & 17.0 & 25 & 5,848 & 7,259 & 8,671 & 10,082 & 11,494 & 12,905 & 14,317 & 15,728 & & \\
\hline 10 & 17.0 & 30 & 5,588 & 6,937 & 8,285 & 9,634 & 10,983 & 12,332 & 13,680 & 15,029 & & \\
\hline 11 & 17.0 & 25 & 5,848 & 7,259 & 8,671 & 10,082 & 11,494 & 12,905 & 14,317 & 15,728 & & \\
\hline 12 & 14.5 & 15 & 6,501 & 8,005 & 9,510 & 11,014 & 12,518 & 14,023 & 15,527 & 17,032 & & \\
\hline 13 & 12.0 & 15 & 6,770 & 8,274 & 9,778 & 11,283 & 12,787 & 14,291 & & & & \\
\hline 14 & 10.0 & 15 & 6,985 & 8,489 & 9,993 & 11,498 & 13,002 & & & & & \\
\hline 15 & & & & & & & & & & & & \\
\hline & & & & & & & & & & & & \\
\hline
\end{tabular}

Legend
\begin{tabular}{|r|l|}
\hline$*$ & About the lip of the hole \\
\hline$* \star$ & Measured at the bottom of the hole \\
\hline$* \star \star$ & Between hole axis and horizontal direction \\
\hline & Chips at the bottom of the hole \\
\hline & Still holding \\
\hline & Breakout \\
\hline
\end{tabular}

Figure 10 - Test results

Tests done on limestone at several angles and depths, testing both the performance of the USDC (power draw, load bearing capability) and that of the rock (load bearing capability), and all requirements were met. The test set-up can be seen in Fig. 9 and the test results are shown in Fig. 10. It can be seen that a 17mm deep hole drilled in Santa Barbara Limestone can support a $16 \mathrm{~N}-\mathrm{m}$ bending moment on a $9 \mathrm{~mm}$ diameter bit.

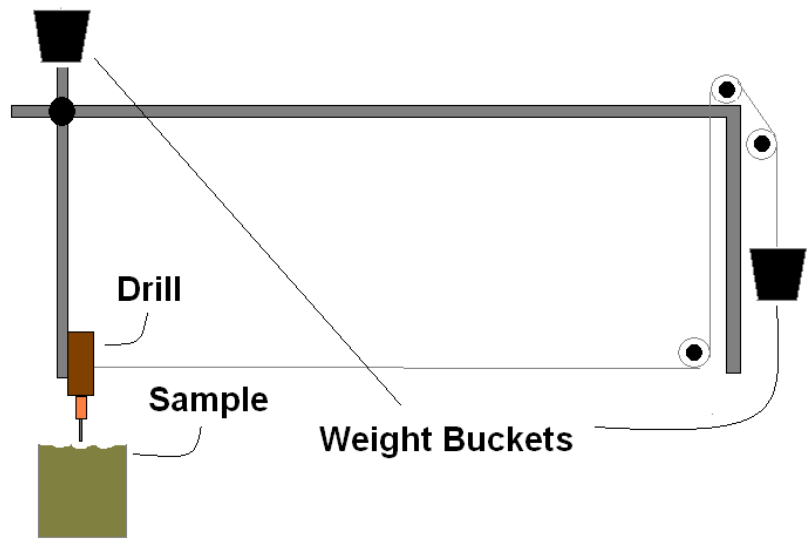

Figure 11 - Loose material testing setup

Drilling needed to be tested in loose materials in order to complete the full spectrum of surfaces that would encounter as part of a mobility system for a robot. The drill was tested in play sand, large gravel $(1.5-2.5 \mathrm{~cm})$, small gravel $(0.5-1.5$ $\mathrm{cm}$ ), and lunar simulant as shown in Fig. 11. Afterwards, it was tested in various mixtures of these materials. The USDC demonstrated that it required less preload for a given penetration depth when active than when passive. Moreover, there was less statistical range for depth vs. preload for active vs. passive operations. The data from these tests will allow for better understanding of the USDC and allow for better mobility design implementation. 


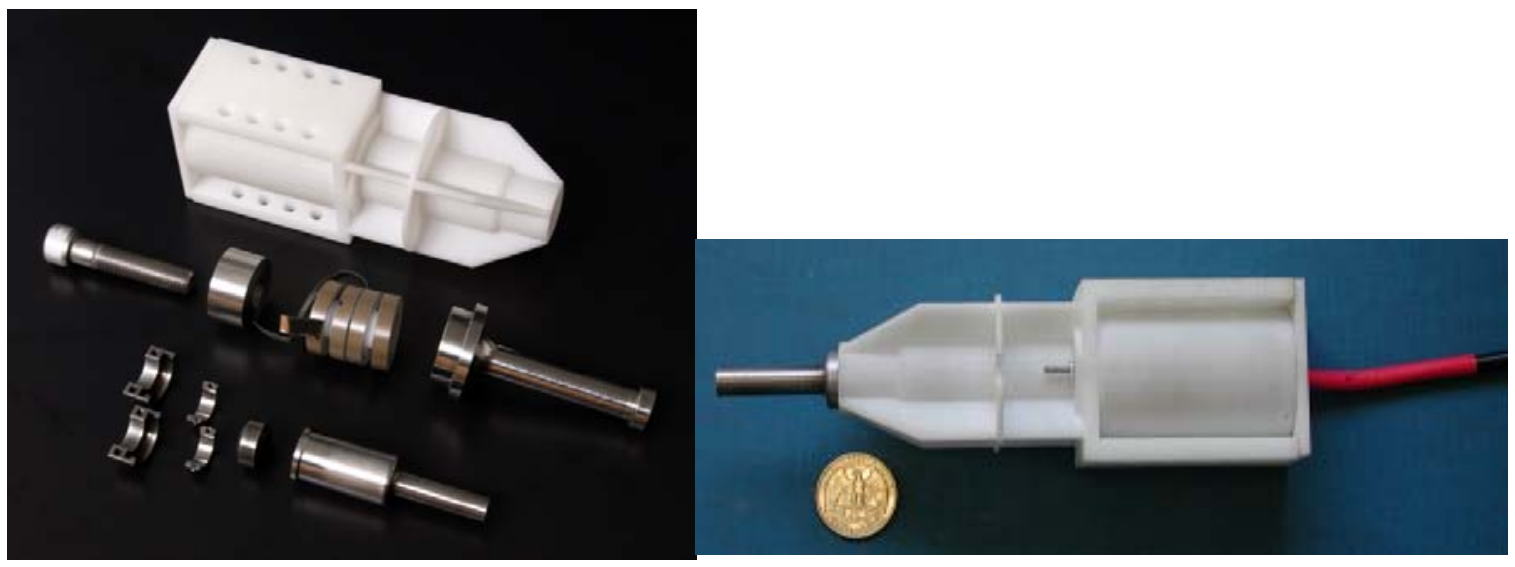

Figure 12 - USDC prototype

The STAR USDC was assembled (see Fig. 12) and qualitatively tested by noting the initial penetration rates in Santa Barbara Limestone. The drilling was notably faster than the original USDC design and the hammering action was noted even when not in contact with the limestone. The STAR USDC was used to drill hole that formed a $30-35^{\circ}$ angle with the normal of the drill surface, was run for one minute continuously the measurements were done as shown in Fig. 13 and the recorded results are shown in Table 1. This adds to 2.5-5 W-hr per hole. It should be noted that for this tests no power optimization has been made. Moreover, the USDC is capable of using duty cycling and so the average power can be decreased significantly. In addition secondary designs with lighter bits may be used to decrease the maximum power. In previous tests we have shown that the USDC can function properly with $30 \mathrm{~W}$ peak power. For this case the total power consumption added to $3 \mathrm{~W}-\mathrm{hr}$ per hole.

Table 1 - Testing results

\begin{tabular}{|c|c|c|c|c|c|}
\hline No. crt. & Duration & Depth & $\operatorname{Angle}(\alpha)$ & Average Power & Peak Power \\
\hline & {$[\mathrm{min}]$} & {$[\mathrm{mm}]$} & $\left.{ }^{\circ}\right]$ & [W] & {$[\mathrm{W}]$} \\
\hline 1 & 1 & 3.5 & 35 & 102.66 & 191.1 \\
\hline 2 & 1 & 3.5 & 30 & 92 & 187.1 \\
\hline 3 & 1 & 4.25 & 32 & 101.93 & 210 \\
\hline 4 & 1 & 5.98 & 30 & 100.17 & 207 \\
\hline
\end{tabular}

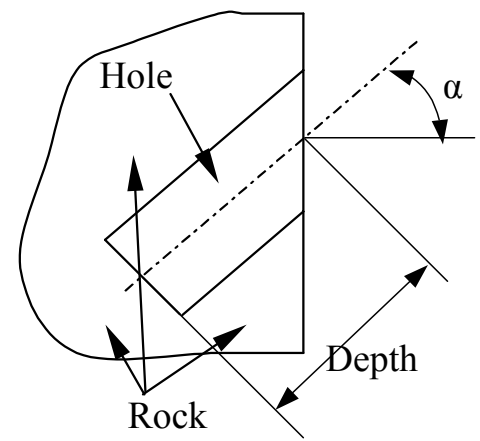

Figure 13 - Drilling measurements

\section{CONCLUDING REMARKS:}

The results of the USDC tests on Santa Barbara limestone suggest that the approach of using the USDC in the feet of a LEMUR class walk robot is feasible and demonstratable. The demonstrated drilling and holding characteristics of the 
USDC bits enables a significant increase in mobility of walking platforms in traversing geological structures. Steep slopes are most often associated with loose material, particularly at their base. The

same action of the USDC that allows it to drill into solid rock also helps the insertion of the entire end-effector into loose material. This fact leads to a mobility mode in which the end-effectors are used like ski poles, digging into the substrate with each step. Such a mode should lead to better mobility on slopes close to the angle of repose.

The STAR design with the forward and reverse hammering action was found to work well and tests are currently being done to quantify the advantage of this design over previous design. The results have been visibly so impressive that we are currently redesigning other USDC systems based on this design to seek improvement in drilling capabilities in other tasks.

\section{ACKNOWLEDGEMENTS}

The research at Jet Propulsion Laboratory (JPL), California Institute of Technology, was funded by the JPL R\&TD program and was carried out under a contract with the National Aeronautics and Space Administration (NASA).

\section{REFERENCES:}

1. Bar-Cohen, Y., Sherrit, S., Dolgin, B., Bao, X., Chang, Z., Krahe, R., Kroh, J., Pal, D., Du, S., Peterson, T., "Ultrasonic/Sonic Driller/Corer (USDC) for planetary application," Proc. SPIE Smart Structure and Materials 2001, pp. 529-551, 2001.

2. P. S. Schenker, P. Pirjanian, B. Balaram, K. S. Ali, A. Trebi-Ollennu, T. L. Huntsberger, H. Aghazarian, B. A. Kennedy and E. T. Baumgartner, Jet Propulsion Laboratory; K. Iagnemma, A. Rzepniewski, and S. Dubowsky, Massachusetts Institute of Technology; P. C. Leger and D. Apostolopoulos, Carnegie Mellon University; G. T. McKee, University of Reading (UK), "Reconfigurable robots for all terrain exploration," Proc. SPIE Vol. 4196, Sensor Fusion and Decentralized Control in Robotic Systems III (Eds. G. T. McKee and P. S. Schenker), 15 pp., Boston, MA, Nov. 5-8, 2000.

3. D. Wettergreen, C. Thorpe, and W.L. Whittaker, "Exploring Mount Erebus by Walking Robot", Robotics and Autonomous Systems, 1993.

4. Wettergreen, D., D. Bapna, M. Maimone and G.Thomas, "Developing Nomad for Robotic Exploration of the Atacama Desert", Robotics \& Autonomous Systems Journal, vol 26, pp. 127-148, 1999.

5. P. Pirjanian, Chris Leger, Erik Mumm, Brett Kennedy, Mike Garrett, Hrand Aghazarian, Shane Farritor, Paul Schenker, "Distributed Control for a Modular, Reconfigurable Cliff Robot", submitted to ICRA 2002.

6. J. Bares and D. Wettergreen, "Dante II: Technical Description, Results and Lessons Learned" International Journal of Robotics Research, Vol. 18, No. 7, July, 1999, pp. 621-649.

7. Y. Bar-Cohen and P. Backes, "Scanning Large Aerospace Structures Using Open-Architecture Crawlers," NSMMS Symposium, San Diego, CA, 27 Feb. to 2 March 2000.

8. Tunstel, E., T. Huntsberger, H. Aghazarian, P. Backes, E. Baumgartner, Y, Cheng, M. Garrett, B. Kennedy, C. Leger, L. Magnone, J. Norris, M. Powell, A. Trebi-Ollennu and P. Schenker, "FIDO Rover Field Trials as Rehearsal for the 2003 Mars Exploration Rover Mission," 9th Intl. Symp. on Robotics \& Applications, 5th World Automation Congress, Orlando, FL, June 2002.

9. R. Volpe, I.A.D. Nesnas, T. Estlin, D. Mutz, R. Petras, H. Das, "The CLARAty Architecture for Robotic Autonomy." Proceedings of the 2001 IEEE Aerospace Conference, Big Sky Montana, March 10-17 2001.

10. S. Sherrit, X. Bao, Z. Chang, B.P. Dolgin, Y. Bar-Cohen, D. Pal, J. Kroh, T. Peterson, "Modeling of the Ultrasonic/Sonic Driller/Corer: USDC," Proceedings of the IEEE Ultrasonics Symposium in San Juan, Puerto Rico, Oct 22-25, 2000

11. X. Bao, Y. Bar-Cohen, Z. Chang, B. P. Dolgin, S. Sherrit, D. S. Pal, S. Du, and T. Peterson, "Modeling and Computer Simulation of Ultrasonic/Sonic Driller/Corer (USDC)," IEEE Transaction on Ultrasonics, Ferroelectrics and Frequency Control (UFFC), Vol. 50, No. 9, (Sept. 2003), pp. 1147-1160.

12. S. Sherrit, M. Badescu, X. Bao, Y. Bar-Cohen, Z. Chang, "Novel Horn Designs for Power Ultrasonics," IEEE International Ultrasonics Symposium, UFFC, Montreal, Canada, August 24-27, 2004. 